(C) 1982. The Genetical Society of Great Britain

\title{
SELECTION IN PLANT POPULATIONS OF EFFECTIVELY INFINITE SIZE: III. THE MAINTENANCE OF FEMALES AMONG HERMAPHRODITES FOR A BIALLELIC MODEL
}

\author{
H.-R. GREGORIUS, M. D. ROSS, and E. GILLET \\ Lehrstuhl für Forstgenetik und Forstpflanzenzüchtung der Universität Göttingen, \\ Büsgenweg 2, 3400 Göttingen-Weende, West Germany
}

Received 26.viii.81

\begin{abstract}
SUMMARY
An allele or genotype is called protected if for all initial genotype frequencies positive it cannot be lost or does not remain at very low frequencies indefinitely. An analysis of protectedness was made for gynodioecious populations (populations with both female and hermaphrodite individuals) with four different types of one-locus two-allele models for the inheritance of gynodioecy. Ovule and pollen fertilities, together with selfing rates may differ between hermaphrodite genotypes. Such factors have not been considered previously, and lead to new conditions for the maintenance of the sex polymorphism. In particular, the mode of genetic control, and the hermaphrodite ovule and pollen fertilities, together with their selfing rates, may determine whether the female genotype is protected. Differential selfing among hermaphrodites may lead to overdominance and allow the maintenance of the polymorphism, and such differential selfing probably occurs in natural gynodioecious populations. In this connection the significance of models of inbreeding depression was considered.
\end{abstract}

\section{INTRODUCTION}

A minority of plant populations contain separate female and hermaphrodite individuals. Such populations are called gynodioecious, and the maintenance of females in these populations is of evolutionary importance because: (a) the females affect the genetic structure of the population; (b) gynodioecy is presumed to have developed from hermaphroditism, despite the lower pollen fertility of females compared to hermaphrodites; and (c) because gynodioecy is often a stage in the evolution of dioecy.

Many authors have studied theoretically the maintenance of females among hermaphrodites (e.g., Lewis, 1941; Jain, 1968; Ross and Shaw, 1971; Valdeyron et al., 1973; Lloyd, 1974; Charnov et al., 1976; Charlesworth and Charlesworth, 1978), and some interesting experimental data are also available (e.g., Lewis and Crowe, 1956; Assouad et al., 1978; Horovitz and Beiles, 1980). However, the theoretical studies are limited to special cases in that they do not allow all the parameters which may be of importance to vary (table 1). For example, none of the models allows hermaphrodite ovule and pollen fertilities to vary ad lib., and only Jain (1968) allows for different selfing rates among hermaphrodites. However, Jain's model applies only to a strain of Origanum vulgare, which shows two-locus inheritance of gynodioecy (Lewis and Crowe, 1956; Jain, 1968). Consequently not even the simple case of one-locus two-allele control of gynodioecy has been exhaustively studied. Furthermore, some theoretically 


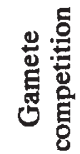
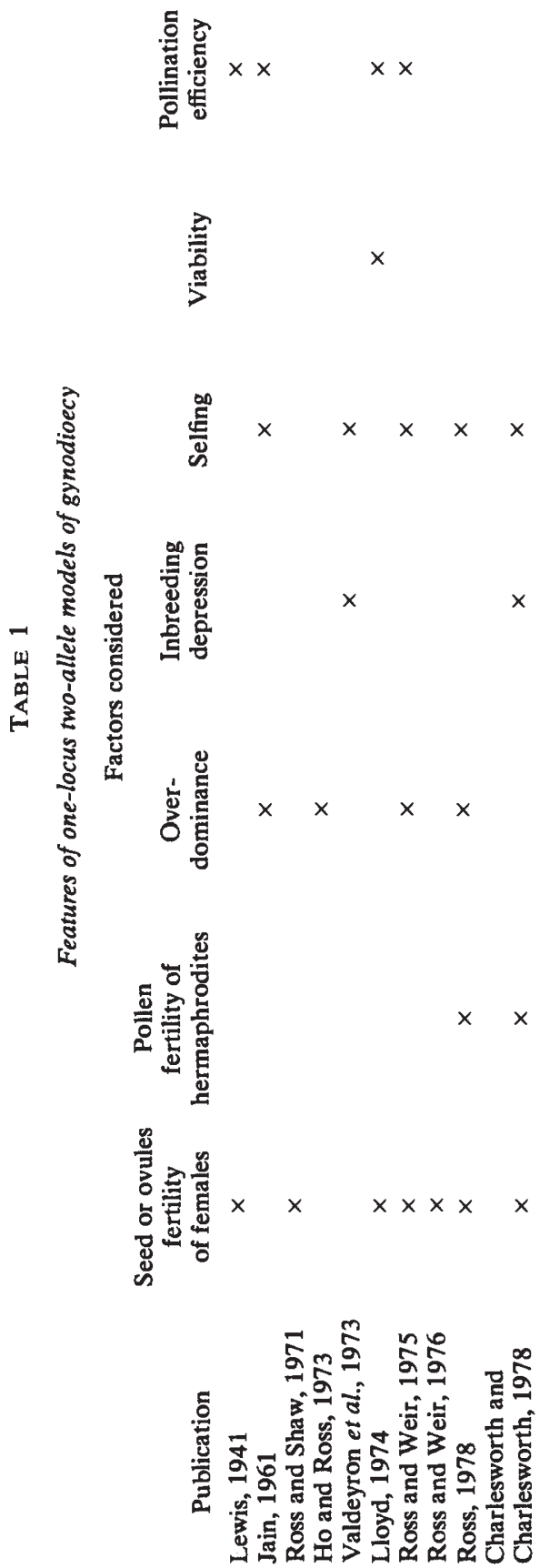
possible assignments of the hermaphrodites and females to the three genotypes of this case have not yet been considered.

The aim of this paper is to make an exhaustive study of one-locus two-allele control of gynodioecy, where every parameter may be varied independently, and where all possible phenotype combinations may be assigned to the three genotypes. Even some apparently unlikely phenotype assignments (e.g., both homozygotes female, heterozygote hermaphrodite) are found to yield interesting insights, and may also be justified by the present limited knowledge of modes of gene action. The present study includes as special cases such previously studied selection mechanisms as overdominance (Jain, 1961, 1968; Ho and Ross, 1973; Ross and Weir, 1975 ) and differences in viability or ovule fertility (e.g., Lewis, 1941; Ross and Shaw, 1971; Lloyd, 1974, 1975). In addition the present study includes new variables, such as fitness differences caused by different selfing rates among hermaphrodites, variation in pollen or ovule fertility among hermaphrodites and sex-asymmetrical fertility differences. Such an analysis has only recently become possible, through the development of the techniques given in the previous paper of this series (Gregorius, 1982). The present paper shows that the genetic control of gynodioecy has a decisive influence on the maintenance of the females. For example, the effects of selfing rates and pollen contributions differ between dominant and recessive models of gynodioecy.

The results of this paper apply to populations containing both female and bisexual individuals, regardless of whether the lattcr are hermaphrodite, where the flowers have organs of both sexes, or monoecious in the botanical sense, where the flowers are unisexual, but where the individuals have flowers of both sexes. The bisexual individuals of gynodioecious populations are, however, usually hermaphrodite (Lloyd, 1976), so that we use the term "hermaphrodite" for the rest of this paper.

\section{DESCRIPTION OF THE MODEL}

The present paper is based on a model introduced in the first two publications of this series. These publications consider a population of effectively infinite size, reproducing in separate generations. An unordered single-locus genotype $A_{i} A_{j}(i, j=1,2)$ is characterized by the number $\phi_{i j}$ of ovules that are produced by an average $A_{i} A_{j}$ plant, the proportion $\sigma_{i j}$ of self-fertilized ovules and the number $\mu_{i j}$ of pollen grains not used in selfing (free pollen). The non-self-fertilized ovules are assumed to be fertilized at random by pollen originating from the population pool of free pollen. $\bar{\phi}$ and $\bar{\mu}$ denote the population averages of the $\phi_{i j}$ 's and $\mu_{i j}$ 's with reference to the frequencies $P_{i j}$ of $A_{i} A_{j}$-zygotes. This leads to the transition equations (primes denote next-generation frequencies)

$$
\begin{aligned}
P_{i i}^{\prime}= & \frac{\sigma_{i i} \phi_{i i} P_{i i}+\frac{1}{4} \sigma_{12} \phi_{12} P_{12}}{\bar{\phi}} \\
& +\frac{\left(1-\sigma_{i i}\right) \phi_{i i} P_{i i}+\frac{1}{2}\left(1-\sigma_{12}\right) \phi_{12} P_{12}}{\bar{\phi}} \cdot \frac{\mu_{i i} P_{i i}+\frac{1}{2} \mu_{12} P_{12}}{\bar{\mu}}
\end{aligned}
$$


for both homozygotes $i=1$ or $i=2$,

for the heterozygote.

$$
\begin{aligned}
P_{12}^{\prime}= & \frac{\frac{1}{2} \phi_{12} P_{12}}{\bar{\phi}}+\frac{\left(1-\sigma_{11}\right) \phi_{11} P_{11}}{\bar{\phi}} \cdot \frac{\mu_{22} P_{22}+\frac{1}{2} \mu_{12} P_{12}}{\bar{\mu}} \\
& +\frac{\left(1-\sigma_{22}\right) \phi_{22} P_{22}}{\bar{\phi}} \cdot \frac{\mu_{11} P_{11}+\frac{1}{2} \mu_{12} P_{12}}{\bar{\mu}}
\end{aligned}
$$

Since, by definition, male genotypes do not exist in gynodioecious populations all $\phi_{i j}$ 's are positive, but for some genotypes, namely the female ones, $\mu_{i j}$ and thus $\sigma_{i j}$ are equal to zero. Therefore, $\mu_{i j}>0$ indicates that $A_{i} A_{j}$ is a hermaphrodite genotype, and according to whether $\sigma_{i j}=1,0<$ $\sigma_{i j}<1$, or $\sigma_{i j}=0$ this genotype self-fertilizes completely, partially or not at all, respectively.

Theoretically there are four different modes of one-locus two-allele control of gynodioecy, which differ with respect to the assignment of the two sexual types to the three genotypes.

(a) $A_{1} A_{1}$ hermaphrodite and both $A_{1} A_{2}$ and $A_{2} A_{2}$ female ( $\mu_{12}=\mu_{22}=$ $\left.\sigma_{12}=\sigma_{22}=0, \mu_{11}>0\right)$. In this case $A_{2}$ may be conceived of as a dominant gene for femaleness, or equivalently, $A_{1}$ as a recessive gene for hermaphroditism.

(b) Both $A_{1} A_{1}$ and $A_{1} A_{2}$ hermaphrodite and $A_{2} A_{2}$ female ( $\mu_{22}=\sigma_{22}=$ $\left.0, \mu_{11}, \mu_{12}>0\right) . A_{2}$ may now be referred to as a recessive gene for femaleness, i.e., $A_{1}$ is a dominant gene for hermaphroditism.

(c) Both homozygotes hermaphrodite, the heterozygote female $\left(\mu_{11}, \mu_{22}>0, \mu_{12}=\sigma_{12}=0\right)$. In this case hermaphroditism shows allelic variation, since, when combined in the heterozygote, the two alleles are "incompatible" with respect to pollen production.

(d) Both homozygotes female and the heterozygote hermaphrodite $\left(\mu_{11}=\mu_{22}=\sigma_{11}=\sigma_{22}=0, \mu_{12}>0\right)$. Femaleness shows allelic variation. Both alleles have a hermaphrodite potential, but in the homozygous state, however, they are "self-incompatible" with respect to pollen production.

The principle of this last model of gene action may be applied to explain all of the four control systems. This principle rests on the idea that both alleles have a hermaphrodite potential per se, but that in the diploid state they may interact in ways which inhibit the expression of the male potential. With respect to pollen production then, in case (a) $A_{2}$ is "incompatible" with itself and with $\boldsymbol{A}_{1}$; in case (b) $\boldsymbol{A}_{2}$ is only "self-incompatible"; in case (c) $A_{1}$ and $A_{2}$ are "incompatible" with each other; and in case (d) both alleles are "self-incompatible". We do not attempt to give detailed biochemical or physiological models for these allele interactions, but recognize that there are many possible explanations.

Our aim is now to derive for each model of gynodioecy precise conditions under which at least one female genotype or all three genotypes are maintained in a population. We understand maintenance in the sense of protectedness of alleles and/or genotypes, and define protectedness as follows:

An allele or genotype is called protected if it cannot be lost from any state of the population where all possible genotypes are present; 
moreover it is required that the allele or genotype cannot remain at arbitrarily low frequencies indefinitely.

The analysis of protectedness as just defined was developed in the preceding paper of this series, for a biallelic polymorphism and for the present model type (Gregorius 1982). Since protectedness of both alleles implies protectedness of a female genotype in all of the above systems of genetic control, this analysis is sufficient for investigating the maintenance of the female phenotype. However, in those cases where the female or hermaphrodite phenotype is expressed by two different genotypes, the question arises as to whether both genotypes or only one genotype can be maintained. This requires an extension of the analysis of protectedness from alleles to genotypes. In order to allow continuity of presentation, the mathematical derivations are deferred to an appendix. The results of these derivations are then discussed for each mode of genetic control of gynodioecy.

\section{Maintenance of the females}

\section{Case (a)}

The Appendix shows that if all three genotypes are present initially, then after the first generation genotype $A_{2} A_{2}$ female is lost. The remaining two genotypes show monotone convergent frequency dynamics such that the female genotype $A_{1} A_{2}$ is protected if and only if $\phi_{12}>2 \phi_{11}$, when the .equilibrium female frequency $\hat{P}_{12}$ equals $\left(\frac{1}{2} \phi_{12}-\phi_{11}\right) /\left(\phi_{12}-\phi_{11}\right)$. Note that $\sigma_{11}$ has no influence on the dynamics, and therefore on the maintenance of the female. This result confirms that of earlier authors (e.g., Lewis 1941; Lloyd 1974; Ross and Weir 1976; Charlesworth and Charlesworth 1978).

\section{Case (b)}

All three genotypes are protected, and hence the monogenic recessive gynodioecy is maintained, if

$$
\frac{1}{2}\left[\phi_{12} / \phi_{11}+\left(1-\sigma_{11}\right) \mu_{12} / \mu_{11}\right]+\frac{1}{4} \sigma_{12} \phi_{12} \phi_{22} / \phi_{11}^{2}>1 .
$$

The gynodioecy is not maintained if the inequality is reversed. This inequality shows that the female is maintained regardless of the selfing rates and pollen fertilities of the hermaphrodites, and regardless of the ovule fertility of the female, if $\phi_{12}>2 \phi_{11}$. For the case $\phi_{12} \leqq 2 \phi_{11}$, the inequality is too complex to easily yield immediate insights, so that it may be useful to study it by varying one parameter at a time. It may also be of interest to apply the inequality to some of the experimental data available in the literature. Thus if the heterozygote does not self-fertilize, $\sigma_{12}=0$, then the maintenance of the female in this case also is essentially independent of its ovule production, since it is sufficient that

$$
\frac{1}{2}\left[\phi_{12} / \phi_{11}+\left(1-\sigma_{11}\right) \mu_{12} / \mu_{11}\right]>1,
$$

and it is necessary that this inequality does not hold in the reverse direction. Therefore, the female is protected if

$$
\sigma_{11}<\frac{2 \mu_{11}}{\mu_{12}}\left(\frac{1}{2}\left(\phi_{12} / \phi_{11}+\mu_{12} / \mu_{11}\right)-1\right)
$$


whereas the female is not protected if the inequality is reversed. This result shows that the selfing rates may play a decisive role in protecting the female genotype $A_{2} A_{2}$ irrespective of its fertility. Clearly, for the above inequality to hold it is necessary that $\frac{1}{2}\left(\phi_{12} / \phi_{11}+\mu_{12} / \mu_{11}\right)>1$. If this condition is not realized, i.e., if $\frac{1}{2}\left(\phi_{12} / \phi_{11}+\mu_{12} / \mu_{11}\right) \leqq 1$, the females may nevertheless be protected if they are sufficiently fertile, namely if

$$
\phi_{22}>\frac{2 \phi_{11}^{2}}{\phi_{12} \cdot \mu_{11}} \cdot\left(2 \mu_{11}-\mu_{12}\right)-2 \phi_{11},
$$

and if both selfing rates are related by the inequality

$$
\sigma_{12}>\frac{4 \phi_{11}^{2}}{\phi_{12} \phi_{22}} \cdot\left[1-\frac{1}{2}\left(\phi_{12} / \phi_{11}+\mu_{12} / \mu_{11}\right)+\sigma_{11} \cdot \frac{1}{2} \mu_{12} / \mu_{11}\right] \text {. }
$$

Given that the fertilities obey (3), fig. 1 provides two examples concerning regions of $\sigma_{11}$ and $\sigma_{12}$ values for which the females are protected.

(a)

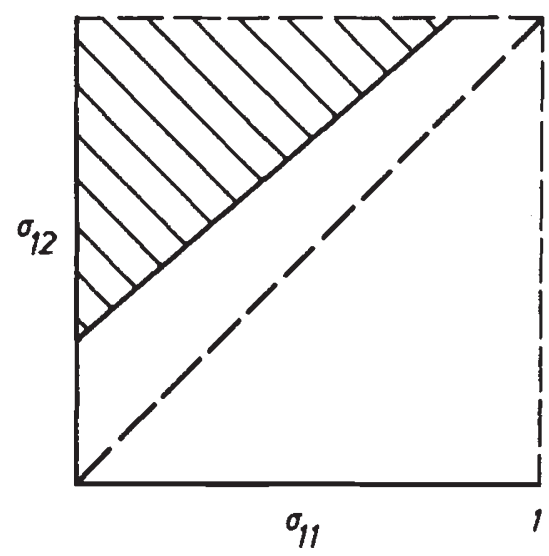

(b)

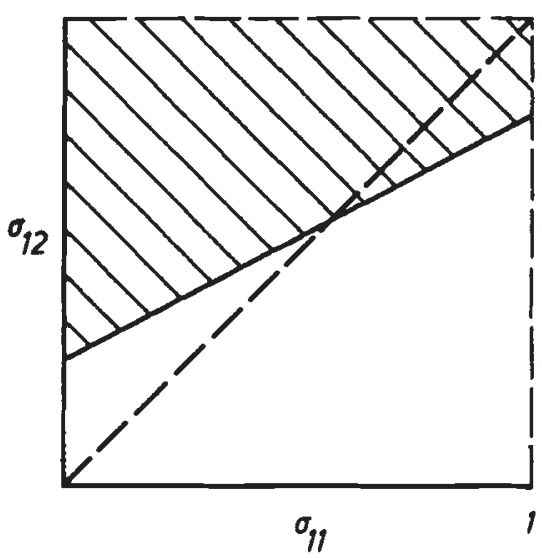

FIG. 1.-Regions (shaded) of hermaphrodite selfing-rates $\sigma_{11}, \sigma_{12}$ guaranteeing female protectedness. (a) Protection impossible for $\sigma_{11}=\sigma_{12}$, (b) protection possible for $\sigma_{11}=$ $\sigma_{12}$.

The figure emphasizes the effects of making the common assumption that the $\sigma$ 's are equal. For equal $\sigma$ 's the set of fertilities applied in fig. 1(a) prevents the establishment of females, whereas the fertilities used in fig. 1(b) allow their establishment, provided the $\sigma$ 's are sufficiently great.

Inequality (2) can also be applied to those species which are both gynodioecious and self-incompatible $\left(\sigma_{11}=\sigma_{12}=0\right)$, such as Plantago lanceolata, some other Plantago species and Hirschfeldia incana (Ross, 1970, 1973; Horovitz and Galil, 1972). In this case females are maintained when

$$
\frac{1}{2}\left(\phi_{12} / \phi_{11}+\mu_{12} / \mu_{11}\right)>1 \text {, }
$$

as is the case $e . g$. , for overdominance for gamete production or for viability (Jain 1961, 1968; Ho and Ross 1974; Ross and Weir 1975). Notice that 
in this case also ovule or seed production of the females has essentially no influence on whether they are protected, since $\sigma_{12}=0$.

Notice that inequality (4) makes no predictions for $\phi_{12}=\phi_{11}, \mu_{12}=\mu_{11}$. Previous studies have shown that females may be maintained by increased seed fertility, under these conditions (e.g., Ross and Weir, 1975). A further study of the effects of selfing rates yields interesting results. For $\phi_{11}=\phi_{12}$ and $\mu_{11}=\mu_{12}$ inequality (2) shows that females are maintained if

$$
\sigma_{12} \phi_{22} / \phi_{11}>2 \sigma_{11} \text {. }
$$

For $\sigma_{12}=\sigma_{11} \neq 0$ this reduces to the previously known result that females may be maintained if they are more than twice as seed-fertile as hermaphrodites (Lewis 1941, and many later authors). But notice that for $\phi_{22}=\phi_{11}$ females may be maintained if the selfing rate of the heterozygous hermaphrodite is more than twice that of the homozygous one, i.e., for $\sigma_{12}>2 \sigma_{11}$. Thus differences in selfing rate may completely replace differences in seed fertility as a mechanism for maintaining females. This result may have the important practical consequence that the mode of maintenance of the females in natural populations may remain undiscovered if the selfing rates of the various hermaphrodite genotypes are not studied. Moreover, there is evidence that selfing rates do indeed differ among hermaphrodites in gynodioecious populations (Lewis and Crowe, 1956; Valdeyron et al., 1977), although only Jain (1968) appears to have recognized the possible significance of this difference for the maintenance of the females. In a strain of Origanum vulgare Lewis and Crowe (1956) found that in an FF or Ff genetic background genotypes $H H$ and $H h$ were hermaphrodite and $h h$ was female. Genotype $H H$ was self-incompatible and $H h$ self-compatible, suggesting that the higher selfing rate of the heterozygote could have a role in maintaining the females, through a form of heterozygote advantage. Although Kheyr-Pour (1981) could not confirm the presence of selfincompatibility in this species, it seems probable that great variations in selfing rates do occur. In the related Thymus vulgaris individual hermaphrodites showed selfing rates which varied from zero to 0.79 , although these rates were not for the sex-control locus itself (Valdeyron et al., 1977). These rates were fairly consistent from year to year, suggesting the presence of a strong genetic component in their determination. Since differential reproductive capacities between females and hermaphrodites do not seem to adequately explain the very high frequency of females sometimes found in Thymus vulgaris (Assouad et al., 1978), it may be desirable to look for over-dominance and differential hermaphrodite genotypic selfing rates in this species. In the present model, differential selfing rates may indeed result in overdominance. For example, for $\phi_{11}=\phi_{12}=\phi_{22}=\phi, \mu_{11}=\mu_{12}=$ $\mu$, and where $P_{11}, P_{12}, P_{22}$ are the frequencies of genotypes $A_{1} A_{1}, A_{1} A_{2}$ and $A_{2} A_{2}$, respectively, we may obtain a fitness value for $A_{1} A_{1}$ as follows: the number of ovule offspring equals $\phi$, the number of pollen offspring from selfing equals $\sigma_{11} \phi$, and the pollen offspring from crossing equals $\bar{\phi}_{(1-\sigma)} \mu / \bar{\mu}$, where $\bar{\phi}_{(1-\sigma)}$ is the mean number of crossed ovules in the population, equals $\phi\left(1-\sigma_{11} P_{11}-\sigma_{12} P_{12}\right)$, and $\bar{\mu}$ is the pollen production, equals $\mu\left(P_{11}+P_{12}\right)$ (Gregorius and Ross, 1981). Thus the total fitness for $A_{1} A_{1}$ equals

$$
\left(1+\sigma_{11}\right) \phi+\frac{\bar{\phi}_{(1-\sigma)}}{P_{11}+P_{12}} .
$$


The fitness of $A_{1} A_{2}$ is obtained in a similar way, and equals

$$
\left(1+\sigma_{12}\right) \phi+\frac{\bar{\phi}_{(1-\sigma)}}{P_{11}+P_{12}},
$$

and the fitness of $A_{2} A_{2}$ equals $\phi$. Clearly $A_{1} A_{2}$ always has the highest fitness value if $\sigma_{12}>\sigma_{11}$, which implies $\sigma_{12}>0$.

For the case of full dominance, $\phi_{11}=\phi_{12}, \mu_{11}=\mu_{12}$ and $\sigma_{11}=\sigma_{12}$, the Appendix shows that the polymorphism is protected if and only if the ovule fertility of the females is more than twice as great as that of the hermaphrodites. Otherwise, fixation of allele $A_{1}$ takes place.

\section{Case (c)}

Here the heterozygote is female, and both homozygotes are hermaphrodite. The Appendix shows that in no case can both hermaphrodites, $A_{1} A_{1}$ and $A_{2} A_{2}$, be protected simultaneously. The biallelic polymorphism, and therefore the gynodioecy, is protected if and only if $\phi_{12}>2 \phi_{11}, 2 \phi_{22}$. This case therefore reduces to case (a) as soon as one of the hermaphrodite genotypes is lost. As in case (a), neither $\sigma$ 's nor $\mu$ 's influence the protectedness of the female. If hermaphrodite genotype $A_{2} A_{2}$ is lost, then allele $A_{2}$ may be regarded as a dominant allele for femaleness, but if hermaphrodite $A_{1} A_{1}$ is lost, then $A_{1}$ is dominant for femaleness. It seems that the apparent absence of this system in nature can just as well be attributed to the impossibility of maintaining both homozygotes simultaneously, as to the seemingly unlikely mode of allele interaction.

During the analysis in the Appendix a particular case arose which may be of interest. This concerns the existence of a continuum of equilibria which constitutes a straight line. This demonstrates that even fairly simple selection models may result in more than one or a few attractive or repulsive equilibria. Another interesting case from the Appendix is that where a population begins as homozygous for hermaphrodite genotype $A_{1} A_{1}$, and then female mutants $A_{1} A_{2}$ become established. Under certain conditions, if some females mutate to give $A_{2} A_{2}$ hermaphrodites, the population can become monomorphic for this last genotype. It is possible, then, for a population to begin as hermaphrodite, become gynodioecious, and then revert to hermaphroditism. This is also an example of why protectedness of an allelic polymorphism must be defined more rigorously than it was originally by Prout (1968).

\section{Case (d)}

In this case both homozygotes are female, and the heterozygote is hermaphrodite. The Appendix shows that after the first generation the hermaphrodites always have frequency 0.5 , and therefore both female genotypes are protected. This system results therefore in the very high female equilibrium frequency of 0.5 , which is greater than in any previously studied nuclear model of gynodioecy (cf. Lloyd, 1974). Note that all possible types of mating yield $1: 1$ ratios if the two female genotypes are indistinguishable, and that this leads to the $1: 1$ sex ratio, as found in dioecy. 
Gregorius (1981) has shown for a general one-locus biallelic model which includes the present one, that fitness values in equilibrium populations are either all equal, or they show underdominance or overdominance. In the present case the fitness values are $\phi_{11}$ for genotype $A_{1} A_{1}, 2 \phi_{12}+$ $\left(\phi_{11} P_{11}+\phi_{22} P_{22}\right) / P_{12}$ for $A_{1} A_{2}$, and $\phi_{22}$ for $A_{2} A_{2}$ (Gregorius and Ross, 1981). In all generations after the first, we may set $P_{12}=0.5$ and $P_{22}=0.5-$ $P_{11}$, so that the fitness of $A_{1} A_{2}$ or $w_{12}$ now equals $2 \phi_{12}-2 P_{11}\left(\phi_{22}-\phi_{11}\right)+$ $\phi_{22}$. If we attempt to set $\phi_{11}=\phi_{22}=w_{12}$ we find that this implies $\phi_{12}=0$, which contradicts the assumptions of this case. Therefore not all fitnesses can be equal after the first generation, or in equilibrium populations. In all generations after the first, if $\phi_{11} \geqq \phi_{22}$ then $w_{12}>\phi_{22}$. If $\phi_{11}<\phi_{22}$, then since $P_{11}<0.5, w_{12}$ has a minimum value of more than $2 \phi_{12}+\phi_{11},>\phi_{11}$. Therefore there can be no underdominance after the first generation. Therefore there can be only overdominance at the equilibrium point, which is shown in the Appendix to be attractive.

\section{Discussion}

Perhaps the most useful feature of the present models is that they consider many possible variables. For example, ovule and pollen fertilities, together with selfing rates, may vary independently between hermaphrodite genotypes. Such variation has the important consequence that the mode of genetic control of gynodioecy may have a decisive influence on the maintenance of the polymorphism. Thus there are important differences in the conditions required for a polymorphism for dominant versus recessive gynodioecy, although both cases have sometimes been considered together (Lloyd, 1975, 1977; Charlesworth and Charlesworth, 1978). Earlier models have shown that females may be maintained if they have more than twice as many ovules or seeds as hermaphrodites, both for monogenic dominant or recessive (Lewis, 1941) or for digenic inheritance (Ross and Shaw, 1971). In the present models this is so only for cases (a) and (c), which essentially refer to dominant gynodioecy. In case (d) maintenance of the females does not depend on their ovule fertility, and in case (b) (recessive gynodioecy) their maintenance may depend upon variation within the hermaphrodites, and not on their ovule fertility (e.g., inequality (3)). In this case a more than doubled seed fertility of the females may not be sufficient for their maintenance.

Several authors (e.g., Valdeyron et al., 1973; Lloyd, 1975; Charlesworth and Charlesworth, 1978) have used a model of inbreeding depression such that offspring of selfings have a lower viability or fertility than offspring of crosses, regardless of offspring genotype. Such a model is genetically imprecise. Strictly speaking, it introduces selective forces which are not genetic, but rather genealogical. However, it is not easy to see how selection may be caused by purely genealogical agencies. Perhaps the intention of those authors was to account for homozygote disadvantage, and they may have used as a justification of their model the frequently observed phenomenon of reduced performance in offspring of selfings. If so, it is equally justified to consider inbreeding depression directly, as a genetic rather than a genealogical force. A study of inbreeding depression may require a multilocus model, and the present study does not aim at such a model. However, if we consider homozygote disadvantage for viability and 
compare the Charlesworths' model (Charlesworth and Charlesworth, 1978) with the present model (b) with complete dominance for the fertility and selfing parameters, we find that the two models give quite distinct results. Expressing inbreeding depression in genetic terms restricted to the locus in question, $\phi_{i i}$ and $\mu_{i i}$ have to be reduced by a factor $1-v$, say, for $i=1,2$. Hence $\phi_{11}=v \phi_{12}, \mu_{11}=v \mu_{12}, \phi_{22}=v / \tilde{\phi}_{22}$ and $\sigma_{11}=\sigma_{12}=\sigma$, where $\phi_{12}$ and $\tilde{\phi}_{22}$ now refer to the ovule fertilities of the hermaphrodites and females, respectively. According to the Appendix the females are protected in case (b) if $\tilde{\phi}_{22}>2 \phi_{12}[1-2(1-v) / \sigma]$. In the Charlesworths' model this condition differs in that $2(1-v) / \sigma$ has to be replaced by $\sigma(1-v)$.

The Charlesworths' model may require more stringent conditions than the present model for the establishment of gynodioecy, as may be seen for example by setting $\tilde{\phi}_{22}=\phi_{12}, v=\frac{1}{2}$, when the Charlesworths' model requires $\sigma>1$ and the present model $\sigma<2$, for the establishment of females. Thus in the first case the condition cannot be fulfilled, whereas in the second it is always fulfilled. Notice that since in the present model the $\sigma$ appears in the denominator of the condition for protectedness, this condition is easier to satisfy with lower $\sigma$, whereas the opposite is true for the Charlesworths' model. Similar situations have been found for overdominant models (Ross and Weir, 1975).

It is hoped to apply similar methods to models of gynodioecy which show both genic and cytoplasmic inheritance, since gene-cytoplasm interactions are frequently found in natural gynodioecious populations.

Acknowledgments. - This work was supported by a Heisenberg fellowship (H.-R.G.) and a grant from the DFG (M.D.R.)

\section{REFERENCES}

ASSOUAD, M. W., DOMMÉE, B., LUMARET, R., AND VALDEYRON, G. 1978. Reproductive capacities in the sexual forms of the gynodioecious species Thymus vulgaris L. Bot. J. Linn. Soc. 77, 29-39.

CHARLESWORTH, B., AND CHARLESWORTH, D. 1978. A model for the evolution of dioecy and gynodioecy. Am. Nat. 112, 975-997.

CHARNOV, E. L., MAYNARD SMITH, J. AND BULL, J. J. 1976. Why be an hermaphrodite? Nature, 263, 125-126.

GREGORIUS, H.-R. 1981. Realized genotypic fitnesses at equilibrium in the deterministic selection theory of a diallelic locus. Göttingen Research Notes in Forest Genetics, 4.

GREGORIUS, H.-R. 1982. Selection in plant populations of effectively infinite size: II. Protectedness of a biallelic polymorphism. $J$. theor. Biol., (in press).

GREGORIUS, H.-R. AND ROSS, M. D. 1981. Selection in plant populations of effectively infinite size: I. Realized genotypic fitnesses. Math. Biosci., 54, 291-307.

HO, T.-Y., AND ROSS, M. D. 1973. Maintenance of male sterility in plant populations. II. Heterotic models. Heredity, 31, 282-286.

HO, T.-Y., AND ROSS, M. D. 1974. Maintenance of males and females in hermaphrodite populations. Heredity 32 113-118.

HOROVITZ, A., AND BEILES, A. 1980. Gynodioecy as a possible populational strategy for increasing reproductive output. Theor. Appl. Genet., 57, 11-15.

HOROVITZ, A., AND GALIL, J. 1972. Gynodioecism in East Mediterranean Hirschfeldia incana. Bot. Gaz., 133, 127-131.

JAIN, S. K. 1961. On the possible adaptive significance of male sterility in predominantly inbreeding populations. Genetics, 46, 1237-1240.

JAIN, S. K. 1968. Gynodioecy in Origanum vulgare: Computer simulation of a model. Nature, $217,764-765$. 
KHEYR-POUR, A. 1981. Wide nucleo-cytoplasmic polymorphism for male sterility in Origanum vulgare L. J. Hered., 72, 45-51.

LEWIS, D. 1941. Male sterility in natural populations of hermaphrodite plants. New Phytol., $40,56-63$.

LEWIS, D., AND CROWE, L. K. 1956. The genetics and evolution of gynodioecy. Evolution, $10,115-125$

LLOYD, D. G. 1974. Theoretical sex ratios of dioecious and gynodioecious Angiosperms. Heredity, 32, 11-34.

LLOYD, D. G. 1975. The maintenance of gynodioecy and androdioecy in Angiosperms. Genetica, 45, 325-339.

LLOYD, D. G. 1976. The transmission of genes via pollen and ovules in gynodioecious Angiosperms. Theor. Popul. Biol., 9, 299-316.

LLOYD, D. G. 1977. Genetic and phenotypic models of natural selection. J. Theor. Biol., 68, 543-560.

PROUT, T. 1968. Sufficient conditions for multiple niche polymorphism. Am. Nat., 102, 493-496.

ROSS, M. D. 1970. Breeding systems in Plantago. Heredity, 25, 129-133.

ROSS. M. D. 1973. Inheritance of self-incompatibility in Plantago lanceolata. Heredity, 30, 169-176.

ROSS, M. D. 1978. The evolution of gynodioecy and subdioecy. Evolution, 32, 174-188.

ROSS, M. D., AND SHAW, R, F. 1971. Maintenance of male sterility in plant populations. Heredity, 26, 1-8.

ROSS, M. D., AND WEIR, B. S. 1975. Maintenance of male sterility in plant populations. III. Mixed selfing and random mating. Heredity, 35, 21-29.

ROSS, M. D., AND WEIR, B. S. 1976. Maintenance of males and females in hermaphrodite populations and the evolution of dioecy, Evolution, 30,425-441.

VALDEYRON, G., DOMMÉE, B., AND VALDEYRON, A. 1973. Gynodioecy: Another computer simulation model. Am. Nat., 107, 454-459.

VALDEYRON, G., DOMMÉE, B., AND VERNET, P. 1977. Self-fertilization in male-fertile plants of a gynodioecious species: Thymus vulgaris L. Heredity, 39, 243-249.

\section{APPENDIX}

\section{Case (a)}

Beginning with a genotypic polymorphic structure $P=\left(P_{11}, P_{12}, P_{22}\right)$, the frequency $P_{22}^{\prime}$ of the female genotype $A_{2} A_{2}$ in the following generation is equal to zero. Thus the female genotype $A_{2} A_{2}$ is not protected. Setting $P_{22}=0$ and $P_{11}=1-P_{12}$ in the general transition equation for $P_{12}$, we obtain

$$
P_{12}^{\prime}=\frac{\frac{1}{2} \phi_{12} P_{12}}{\phi_{11}+\left(\phi_{12}-\phi_{11}\right) P_{12}} \text {. }
$$

If $\phi_{11} \geqq \frac{1}{2} \phi_{12}$, then all structures converge to the fixed point $\hat{P}_{12}:=0$, and thus the female genotype $A_{1} A_{2}$ as well as the allele $A_{2}$ are not protected. If $\phi_{11}<\frac{1}{2} \phi_{12}$, then all structures converge to

$$
\hat{P}_{12}:=\frac{\frac{1}{2} \phi_{12}-\phi_{11}}{\phi_{12}-\phi_{11}}>0,
$$

so that the female $A_{1} A_{2}$ and thus the allelic polymorphism are protected. Hence, the female $A_{1} A_{2}$ is protected if and only if $\phi_{11}<\frac{1}{2} \phi_{12}$.

\section{Case (b)}

It is immediately clear that protectedness of the female genotype $A_{2} A_{2}$ and the allele $A_{2}$ are equivalent. It follows from Gregorius (1982), Section 
3, that $A_{2}$ is protected if $\hat{\theta}_{2}>1$, where

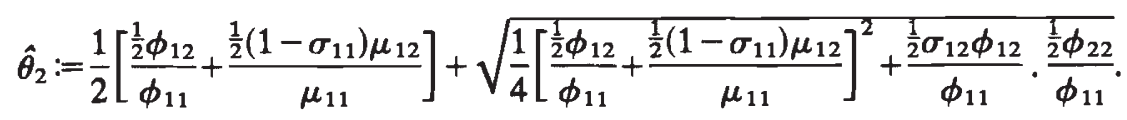

Since $\hat{\theta}_{2} \geqq \frac{1}{2}\left[\phi_{12} / \phi_{11}+\left(1-\sigma_{11}\right) \mu_{12} / \mu_{11}\right], A_{2}$ is protected if $\frac{1}{2}\left(\phi_{12} / \phi_{11}+\right.$ $\left.\mu_{12} / \mu_{11}\right)>1+\frac{1}{2} \sigma_{11} \mu_{12} / \mu_{11}$. This is also the exact condition if $\sigma_{12}=0$. Otherwise, i.e., if $\frac{1}{2}\left(\phi_{12} / \phi_{11}+\left(1-\sigma_{11}\right) \mu_{12} / \mu_{11}\right) \leqq 1, \hat{\theta}_{2}>1$ is equivalent to

$$
\begin{aligned}
& \frac{1}{4}\left[\frac{1}{2} \phi_{12} / \phi_{11}+\frac{1}{2}\left(1-\sigma_{11}\right) \mu_{12} / \mu_{11}\right]^{2}+\frac{1}{4} \sigma_{12} \phi_{12} \phi_{22} / \phi_{11}^{2} \\
&> {\left[1-\frac{1}{2}\left(\frac{1}{2} \phi_{12} / \phi_{11}+\frac{1}{2}\left(1-\sigma_{11}\right) \mu_{12} / \mu_{11}\right]^{2}\right.} \\
&= 1-\frac{1}{2}\left(\phi_{12} / \phi_{11}+\left(1-\sigma_{11}\right) \mu_{12} / \mu_{11}\right) \\
&+\frac{1}{4}\left[\frac{1}{2} \phi_{12} / \phi_{11}+\frac{1}{2}\left(1-\sigma_{11}\right) \mu_{12} / \mu_{11}\right]^{2},
\end{aligned}
$$

which is in turn equivalent to

$$
1<\frac{1}{2}\left(\phi_{12} / \phi_{11}+\left(1-\sigma_{11}\right) \mu_{12} / \mu_{11}\right)+\frac{1}{4} \sigma_{12} \frac{\phi_{12} \phi_{22}}{\phi_{11}^{2}} .
$$

Hence,

$$
\hat{\theta}_{2}>1 \Leftrightarrow \frac{\frac{1}{2} \phi_{12}}{\phi_{11}}+\frac{\frac{1}{2}\left(1-\sigma_{11}\right) \mu_{12}}{\mu_{11}}+\frac{1}{4} \sigma_{12} \frac{\phi_{12} \phi_{22}}{\phi_{11}^{2}}>1 .
$$

For the special case of complete dominance of the allele $A_{1}$ for all parameters, i.e., $\phi_{11}=\phi_{12}=: \phi, \mu_{11}=\mu_{12}=: \mu$, and $\sigma_{11}=\sigma_{12}=: \sigma$, the frequency $p_{1}^{\prime}$ of the allele $A_{1}$ in the next generation reads

$$
p_{1}^{\prime}=p_{1} \cdot \frac{\phi\left(P_{11}+P_{12}\right)+\frac{1}{2} \phi_{22} P_{22}}{\left(P_{11}+P_{12}\right)\left(\phi\left(P_{11}+P_{12}\right)+\phi_{22} P_{22}\right)} \text {. }
$$

In this case, a necessary and sufficient condition for protectedness of $\boldsymbol{A}_{2}$ can be obtained by considering the following relationship:

$$
\begin{aligned}
\frac{p_{1}}{p_{1}^{\prime}} & =\left(1-P_{22}\right) \cdot\left[1+\frac{\frac{1}{2} \phi_{22} P_{22}}{\frac{1}{2} \phi_{22} P_{22}+\phi\left(1-P_{22}\right)}\right] \\
& =1+P_{22} \cdot \frac{\left(1-P_{22}\right)\left(\frac{1}{2} \phi_{22}-\phi\right)-\frac{1}{2} \phi_{22} P_{22}}{\frac{1}{2} \phi_{22} P_{22}+\phi\left(1-P_{22}\right)} .
\end{aligned}
$$

It follows immediately that for $\frac{1}{2} \phi_{22} \leqq \phi, p_{1} / p_{1}^{\prime}<1$, which in turn implies fixation of $A_{1}$. If $\frac{1}{2} \phi_{22}>\phi$, the fraction in the above equation must be positive for small values of $P_{22}$, hence $p_{1}^{\prime}<p_{1}$ for $p_{2}$ sufficiently small. This, however, is tantamount to protectedness of $\boldsymbol{A}_{2}$ and therefore of the female $A_{2} A_{2}$. It is also the same condition as in (a).

\section{Case (c)}

We begin with a genotypic polymorphic structure $P=\left(P_{11}, P_{12}, P_{22}\right)$. Letting $i, j \in\{1,2\}, i \neq j$, and again applying the results of Gregorius (1982), the allele $\boldsymbol{A}_{i}$ is protected if $\hat{\theta}_{i}>1$, and it is not protected if $\hat{\theta}_{i}<1$, where

$$
\hat{\theta}_{i}=\max \left\{\sigma_{i i} \phi_{i i}, \frac{1}{2} \phi_{12}\right\} / \phi_{i j}
$$


It follows that $\boldsymbol{A}_{1}$ and $\boldsymbol{A}_{2}$ are both protected if $\hat{\boldsymbol{\theta}}_{1}, \hat{\boldsymbol{\theta}}_{2}>1$ or, equivalently,

$$
\max \left\{\phi_{11}, \phi_{22}\right\}<\frac{1}{2} \phi_{12} \text {. }
$$

Since the female genotype $A_{1} A_{2}$ is protected if and only if both alleles are protected, this is the condition for the maintenance of the female, and it is essentially independent of selfing and pollen production.

In the other direction, if the allelic polymorphism is protected, then $\hat{\theta}_{1}, \hat{\theta}_{2} \geqq 1$. This is the case if and only if one of the following holds:

$$
\begin{aligned}
& \text { (i) } \max \left\{\phi_{11}, \phi_{22}\right\} \leqq \frac{1}{2} \phi_{12} \\
& \text { (ii) } \phi_{11}=\phi_{22}>\frac{1}{2} \phi_{12} \text { and } \sigma_{11}=\sigma_{22}=1 \text {. }
\end{aligned}
$$

In case (ii), the transition equation for $P_{12}$ reads

$$
P_{12}^{\prime}=P_{12} \cdot \frac{\frac{1}{2} \phi_{12}}{\bar{\phi}}
$$

Since $\frac{1}{2} \phi_{12}<\bar{\phi}$ for all structures, $P_{12}^{\prime}<P_{12}$. Therefore, $P_{12}$ converges to zero and the allelic polymorphism is not protected. Hence, (i) is necessary for protectedness of the allelic polymorphism.

The homozygote $A_{i} A_{i}, i \in\{1,2\}$, can be lost only by convergence of the genotypic structure to an equilibrium point located on the boundary $P_{i i}=0$. The fixation points $P_{11}=1$ and $P_{22}=1$ are always equilibrium points. As was shown in case (a) of this appendix, (i) is the condition for the existence of additional equilibrium points $\hat{P}_{1}$ and $\hat{P}_{2}$ on the boundary $P_{11}=0$ and $P_{22}=0$, respectively, where for $i, j \in\{1,2\}, i \neq j, \hat{P}_{i}$ is defined by

$$
\hat{P}_{i i}=0, \quad \hat{P}_{12}=\frac{\frac{1}{2} \phi_{12}-\phi_{i j}}{\phi_{12}-\phi_{i j}}, \quad \hat{P}_{i j}=1-\hat{P}_{12} .
$$

(If $\phi_{i j}=\frac{1}{2} \phi_{12}$, then $\hat{P}_{i}$ is the point $P_{i j}=1$.) Letting $P$ denote structures with all genotypes present,

$$
\frac{P_{i i}^{\prime}}{P_{i i}} \underset{P \rightarrow P_{i}}{\longrightarrow} \frac{\sigma_{i i} \phi_{i i} \mu_{j i}+\mu_{i i}\left(\frac{1}{2} \phi_{12}-\phi_{i j}\right)}{\frac{1}{2} \phi_{12} \mu_{i j}}=: G_{i i} \text {. }
$$

If $G_{i i}<1$, then $\hat{P}_{i}$ is attractive, and thus $A_{i} A_{i}$ is not protected; if $G_{i i}>1$, then $\hat{P}_{i}$ is repulsive.

Assume that under condition (i) $\phi_{j j}=\frac{1}{2} \phi_{12}$, i.e., $\phi_{i l} \leqq \frac{1}{2} \phi_{12}=\phi_{j j}$. Then $\hat{P}_{i}$ is the point $P_{j i}=1$, and $G_{i i} \leqq 1$ with equality holding if and only if $\sigma_{i i}=1$ and $\phi_{i i}=\frac{1}{2} \phi_{12}$. Therefore, if $\phi_{i l}<\frac{1}{2} \phi_{12}$ or $\sigma_{i i}<1$, then $\hat{P}_{i}$ is attractive and the allele $A_{i}$ is not protected. Furthermore, if $\phi_{11}=\frac{1}{2} \phi_{12}=\phi_{22}$ and $\sigma_{11}=$ $\sigma_{22}=1$, then

$$
P_{12}^{\prime}=P_{12} \cdot \frac{1}{1+P_{12}}<P_{12}
$$

and, hence, $P_{12}$ converges to zero. Therefore, we have a further reduction in the necessary condition for the protectedness of the allelic polymorphism. It now reads

$$
\max \left\{\phi_{11}, \phi_{22}\right\}<\frac{1}{2} \phi_{12} \text {. }
$$

The protectedness of the allelic polymorphism is guaranteed by the condition. Since each of the boundaries $P_{11}=0$ and $P_{22}=0$ has a unique 
equilibrium point $\left(\hat{P}_{1}\right.$ resp. $\left.\hat{P}_{2}\right)$ in its interior (see case (a)), the protectedness of both homozygotes is equivalent to the repulsivity of $\hat{P}_{1}$ and $\hat{P}_{2}$. The repulsivity of $\hat{P}_{1}$ and $\hat{P}_{2}$ implies $G_{11}, G_{22} \geqq 1$, which is in turn equivalent to

$$
\mu_{i i}\left(\frac{1}{2} \phi_{12}-\phi_{i j}\right) \geqq u_{i j}\left(\frac{1}{2} \phi_{12}-\sigma_{i i} \phi_{i i}\right)
$$

for both pairs $i, j \in\{1,2\}, i \neq j$. Since all terms are positive, this results in

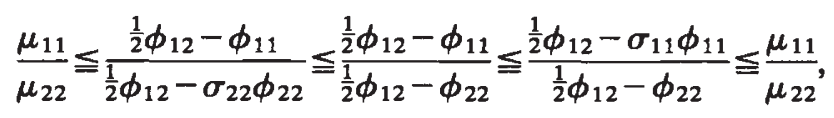

which implies

$$
\sigma_{11}=\sigma_{22}=1 \text { and } \frac{\mu_{11}}{\mu_{22}}=\frac{\frac{1}{2} \phi_{12}-\phi_{11}}{\frac{1}{2} \phi_{12}-\phi_{22}} .
$$

The transition equation for $P_{12}$ reads

$$
P_{12}^{\prime}=P_{12} \cdot \frac{\frac{1}{2} \phi_{12}}{\bar{\phi}}
$$

and, therefore, it holds that $P_{12}^{\prime}=P_{12}$, i.e., $\frac{1}{2} \phi_{12}=\bar{\phi}$, on the straight line connecting $\hat{P}_{1}$ and $\hat{P}_{2}$.

Setting $P_{12}^{\prime}=P_{12}$ and rearranging, the equation of this line is

$$
P_{12}=\frac{\frac{1}{2} \phi_{12}-\phi_{i i}}{\phi_{12}-\phi_{i i}}+P_{i i} \cdot \frac{\phi_{i j}-\phi_{i i}}{\phi_{12}-\phi_{i j}} .
$$

To see that all points on this line are fixed points, insert

$$
\frac{\mu_{i i}}{\mu_{i j}}=\frac{\frac{1}{2} \phi_{12}-\phi_{i i}}{\frac{1}{2} \phi_{12}-\phi_{i j}}
$$

or, equivalently,

$$
\frac{\mu_{i i}}{\bar{\mu}}=\frac{\frac{1}{2} \phi_{12}-\phi_{i i}}{\frac{1}{2} \phi_{12}\left(1+P_{12}\right)-\bar{\phi}}
$$

into the transition equation for $P_{i i}$.

Considering that on this line by definition $\frac{1}{2} \phi_{12}=\bar{\phi}$, the result follows immediately. Hence, in the vicinity of the fixed point $P_{i}$, at least all points on the above line do not fulfill the second part of the definition of the protectedness of $A_{i} A_{i}$.

Consequently, it is not possible to have both homozygotes protected simultaneously. Furthermore, the analysis shows that the biallelic polymorphism is protected if and only if $\max \left\{\phi_{11}, \phi_{22}\right\}<\frac{1}{2} \phi_{12}$.

\section{Case (d)}

Beginning with a genotypic polymorphic structure $P=\left(P_{11}, P_{12}, P_{22}\right)$, the structure in the next generation is given by

$$
P_{i i}^{\prime}=\frac{\frac{1}{2} \phi_{i i} P_{i i}+\frac{1}{4} \phi_{12} P_{12}}{\phi_{i i} P_{i i}+\phi_{12} P_{12}+\phi_{i l} P_{i j}} \text { for } i, j \in\{1,2\}, \quad i \neq j,
$$


and $P_{12}^{\prime}=\frac{1}{2}$. Therefore the heterozygote and thus the genotypic polymorphism are always maintained. The rates of self-fertilization and pollen production have no influence on the genotypic structure.

For $P_{12}=\frac{1}{2}, P_{i i}^{\prime}$ is a strictly increasing convex or concave function of $P_{i i}$ with $P_{i i}^{\prime}>0$ for $P_{i i}=0$ and $P_{i i}^{\prime}<\frac{1}{2}$ for $P_{i i}=\frac{1}{2}$. Consequently, after the first generation montone convergence to a unique equilibrium takes place. 